\title{
Fetal porcine mesenchymal stem cells nuclear transfer and isolation of embryonic stem cells-like derived from cloned blastocysts
}

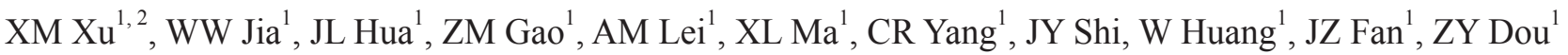 \\ ${ }^{1}$ Northwest A \& F University, Shaanxi Branch of National Stem cell Engineering and Technology, P.O. Box 1-10, Shaanxi Yangling \\ 712100, China; ${ }^{2}$ National Center of Human stem cell Research and Engineering, Institute of Human Reproduction and Stem Cell \\ Engineering, Central South University, Changsha 410008, China
}

The present study was conducted to establish a mesenchymal stem cells (MSCs) line from porcine fetal bone marrow and compare the efficiency of nuclear transfer using porcine fetal mesenchymal stem cells (pFMSCs), porcine fetal fibroblasts (pFF) and newborn porcine ear skin fibroblasts (pESF) three types donor cell respectively, as well as initially explore the possibility of isolation and culture of ESCs-like from cloned blastocysts. A pFMSCs line was successfully established and expanded up to at least passage 13, which still presented a stable fibroblastlike morphology. Flow cytometry analysis confirmed that pFMSCs expressed CD44, CD105 and CD117 rather than CD45, CD11a and CD14. pFF0306, pESF109 and pFMSCs0310 were used as donor cells to perform SCNT respectively, and there were no significant differences in fusion rate, cleavage rate and blastocysts formation rate $(P>$ 0.05). PZM-3, compared with NCSU-23, can increase the cleavage rate $(61.4 \%$ vs $57.6 \%)$ and blastocyst rate $(15.9 \%$ vs $9.0 \%$ ) of porcine SCNT embryo, but there were no significant differences in statistics. Insulin and EGF added to the PZM-3 significantly increased the total cell number of blastocysts $(P<0.05)$. Cloned embryos were transferred to four recipients, two of these became pregnant, and one gave birth to a cloned dead fetuses derived from pFF0306 donor cells at pregnant day 121. Sequence analysis demonstrated the DNA sequence of the cloned fetus was identical to that of the donor cells and not genetically related to the recipient gilt. ESCs-like colonies were successfully isolated from cloned blastocysts and these cells showed AP activity and could be maintained for up to 1 passage. The results of the present study indicate that reconstructed embryos have the ability to maintain pregnancy to 51 day in vivo and ESCs-like can be derived from cloned blastocysts in vitro by transferring the pFMSCs into enucleated oocytes.

Keywords: somatic cell nuclear transfer, mesenchymal stem cells, embryonic stem cells, porcine

Cell Research (2008) 18:s143. doi: 10.1038/cr.2008.233; published online 4 August 2008

Correspondence: Xiaoming Xu

E-mail: xiaomingxu613@126.com 